\title{
LSO:Ce Inorganic Scintillators Are Biocompatible With Neuronal and Circuit Function
}

\begin{abstract}
Aundrea F. Bartley ${ }^{1,2,3,4 \dagger}$, Kavitha Abiraman ${ }^{4,5 \dagger}$, Luke T. Stewart ${ }^{4,5}$, Mohammed lqbal Hossain ${ }^{6}$, David M. Gahan ${ }^{1}$, Abhishek V. Kamath ${ }^{1}$, Mary K. Burdette ${ }^{7}$, Shaida Andrabe ${ }^{6}$, Stephen H. Foulger ${ }^{7,8,9}$, Lori L. McMahon ${ }^{2,3,4,5 *}$ and Lynn E. Dobrunz ${ }^{1,2,3,4 *}$

${ }^{1}$ Department of Neurobiology, University of Alabama at Birmingham, Birmingham, AL, United States, ${ }^{2}$ Civitan International Research Center, University of Alabama at Birmingham, Birmingham, AL, United States, ${ }^{3}$ Evelyn F. McKnight Brain Institute, University of Alabama at Birmingham, Birmingham, AL, United States, ${ }^{4}$ Comprehensive Neuroscience Center, University of Alabama at Birmingham, Birmingham, AL, United States, ${ }^{5}$ Department of Cell, Developmental, and Integrative Biology, University of Alabama at Birmingham, Birmingham, AL, United States, ${ }^{6}$ Department of Pharmacology, University of Alabama at Birmingham, Birmingham, AL, United States, ${ }^{7}$ Department of Materials Science and Engineering, Clemson University, Anderson, SC, United States, ${ }^{8} \mathrm{Center}$ for Optical Materials Science and Engineering Technologies, Clemson University, Anderson, SC, United States, ${ }^{9}$ Department of Bioengineering, Clemson University, Clemson, SC, United States
\end{abstract}

\section{OPEN ACCESS}

Edited by:

Carl R. Lupica, National Institute on Drug Abuse (NIDA), United States

Reviewed by: Carlos Fernando Valenzuela, University of New Mexico, United States

Laura Schrader,

Tulane University, United States

*Correspondence: Lori L. McMahon mcmahon@uab.edu Lynn E. Dobrunz dobrunz@uab.edu

tThese authors have contributed equally to this work

Received: 22 March 2019 Accepted: 06 August 2019 Published: 03 September 2019

Citation: Bartley AF, Abiraman K, Stewart LT, Hossain MI, Gahan DM, Kamath AV, Burdette MK, Andrabe S, Foulger SH, MCMahon LL and Dobrunz LE (2019) LSO:Ce Inorganic Scintillators Are Biocompatible With Neuronal and Circuit Function. Front. Synaptic Neurosci. 11:24. doi: 10.3389/fnsyn.2019.00024
Optogenetics is widely used in neuroscience to control neural circuits. However, non-invasive methods for light delivery in brain are needed to avoid physical damage caused by current methods. One potential strategy could employ $\mathrm{x}$-ray activation of radioluminescent particles (RPLs), enabling localized light generation within the brain. RPLs composed of inorganic scintillators can emit light at various wavelengths depending upon composition. Cerium doped lutetium oxyorthosilicate (LSO:Ce), an inorganic scintillator that emits blue light in response to x-ray or ultraviolet (UV) stimulation, could potentially be used to control neural circuits through activation of channelrhodopsin-2 (ChR2), a light-gated cation channel. Whether inorganic scintillators themselves negatively impact neuronal processes and synaptic function is unknown, and was investigated here using cellular, molecular, and electrophysiological approaches. As proof of principle, we applied UV stimulation to $4 \mu \mathrm{m} \mathrm{LSO}$ :Ce particles during whole-cell recording of CA1 pyramidal cells in acute hippocampal slices from mice that expressed ChR2 in glutamatergic neurons. We observed an increase in frequency and amplitude of spontaneous excitatory postsynaptic currents (sEPSCs), indicating activation of ChR2 and excitation of neurons. Importantly, LSO:Ce particles did not affect survival of primary mouse cortical neurons, even after $24 \mathrm{~h}$ of exposure. In extracellular dendritic field potential recordings, no change in the strength of basal glutamatergic transmission was observed during exposure to LSO:Ce microparticles. However, the amplitude of the fiber volley was slightly reduced with high stimulation. Additionally, there was a slight decrease in the frequency of sEPSCs in whole-cell voltage-clamp recordings from CA1 pyramidal cells, with no change in current amplitudes. The amplitude and frequency of spontaneous inhibitory postsynaptic currents were unchanged. Finally,

\footnotetext{
Abbreviations: aCSF, artificial cerebral spinal fluid; ChR2, channelrhodopsin-2; fEPSP, field excitatory postsynaptic potentials; GFAP, glial fibrillary acidic protein; LED, light emitting diodes; LSO:Ce, Cerium doped lutetium oxyorthosilicate; LTP, long term potentiation; PBS, phosphate buffered saline; RLP, radioluminescent particle; sEPSCs, spontaneous excitatory postsynaptic currents; sIPSCs, spontaneous inhibitory postsynaptic currents; UV, ultraviolet.
} 
long term potentiation (LTP), a synaptic modification believed to underlie learning and memory and a robust measure of synaptic integrity, was successfully induced, although the magnitude was slightly reduced. Together, these results show LSO:Ce particles are biocompatible even though there are modest effects on baseline synaptic function and long-term synaptic plasticity. Importantly, we show that light emitted from LSO:Ce particles is able to activate ChR2 and modify synaptic function. Therefore, LSO:Ce inorganic scintillators are potentially viable for use as a new light delivery system for optogenetics.

\section{Keywords: radioluminescent, scintillator, optogenetics, biocompatible, LTP, LSO:Ce}

\section{INTRODUCTION}

Over the past decade, the field of optogenetics has expanded our knowledge about the role of individual neuronal cells and specific brain circuits in behavior and disease states (Gradinaru et al., 2009; Yizhar et al., 2011; Lim et al., 2013; Gunaydin et al., 2014; Emiliani et al., 2015; Fenno et al., 2015; Rost et al., 2017; Selimbeyoglu et al., 2017; Barnett et al., 2018). Optogenetics relies on the expression of exogenous light-activated ion channels, including the blue light-activated channelrhodopsin-2 (ChR2), that causes depolarization, or the orange-light activated halorhodopsin, which causes hyperpolarization, of the membrane potential of brain cells of interest. Despite these great advances, improvements to the method are constantly being developed (Rein and Deussing, 2012; Lim et al., 2013; Lin et al., 2017; Chen et al., 2018). The use of optogenetics in vivo requires delivery of light into the brain, which is most commonly done through implantation of fiber optic waveguides (fibers) or light emitting diodes (LEDs). These can be as large as several 100 microns, which causes damage to delicate brain tissues, especially when implanted deep within brain structures (Aravanis et al., 2007; Ozden et al., 2013; Canales et al., 2018). In addition, attenuation of light occurs through absorption and scattering in brain tissue, resulting in the need for higher intensities of light at the source. This illumination often results in enhanced local temperatures, a consequence that is greater with stronger and more frequent irradiance (Senova et al., 2017), and can lead to as much as a $30 \%$ increase in local neuron firing rates (Stujenske et al., 2015). Lastly, glial scarring can occur at the light source (Podgorski and Ranganathan, 2016), decreasing effective light intensities and leading to variability in neuronal control. Currently, there are very few options for noninvasive methods of light delivery into the brain for optogenetics.

Efforts are ongoing to develop and refine minimally invasive strategies for the generation of light within the brain to combat the challenges mentioned above (Chen et al., 2018). One potential strategy could employ the use of $\mathrm{x}$-rays to activate radioluminescent materials (Berry et al., 2015; French et al., 2018; Shuba, 2019). Radioluminescence is the production of visible light by a material exposed to ionizing radiation. Radioluminescent particles (RLPs) can be generated from inorganic scintillator material, which would emit light at different wavelengths depending upon its composition. RLP technology could be superior to current invasive methods because it obviates the need for implanting devices into brain tissue that can cause damage and lose effectiveness over time. In addition, the light will be generated locally and have less attenuation due to tissue scattering. This should allow for much lower power densities needed to achieve opsin activation, thereby reducing nonspecific heat-related effects to the tissue. Finally, the uniformity of light delivery will reduce non-specific effects of a graded response due to light absorption and scatter from a single point source. However, it is unknown whether these inorganic RLPs themselves will impact neuronal processes and synaptic function.

One of the most common inorganic scintillators used in medical imaging is Cerium-doped lutetium oxyorthosilicate (LSO:Ce). LSO:Ce crystals are used as detectors in medical imaging devices because they absorb $\mathrm{x}$-rays and $\gamma$-rays extremely well (high density of $7.41 \mathrm{~g} / \mathrm{cm}^{3}$ ), have a high light output $(\sim 30,000$ photons/MeV) and extremely fast decay kinetics ( $\sim 40$ ns; Melcher and Schweitzer, 1992; Roy et al., 2013). The polycrystalline powder form of LSO:Ce has similar properties to the single crystal (Lempicki et al., 2008), and would be within the appropriate size range to be used as a RLP in less invasive optogenetic control of brain circuits. Importantly for optogenetics, activation of LSO:Ce by x-rays emits light in the appropriate range to activate ChR2 (Melcher and Schweitzer, 1992). Since it is a rare-earth metal, it is considered to have a low degree of toxicity. However, little is known about the effect of LSO:Ce particles on neurons, which are extremely sensitive to their environment.

Here, we demonstrate that LSO:Ce particles, applied for $24 \mathrm{~h}$, had no effect on neuronal survival in primary cortical cultures. Using hippocampal brain slices, LSO:Ce particles did not alter basal synaptic excitatory field potentials (fEPSPs) at CA3-CA1 synapses, however, there was a slight decrease in the fiber volley. The frequency of spontaneous excitatory post-synaptic currents (sEPSCs) in CA1 pyramidal cells was slightly reduced. LSO:Ce microparticles did not impair the induction of long term potentiation (LTP), although there was a slight decrease in LTP amplitude. Importantly, we also demonstrate that light emitted from LSO:Ce microparticles following irradiation is able to activate ChR2 and modulate synaptic function. Together, these results show that LSO:Ce particles are not overtly toxic to neurons or brain slices, although there are modest effects on baseline synaptic function and plasticity. Therefore, the LSO:Ce 
particles are a potentially viable tool for a less invasive form of in vivo optogenetics.

\section{MATERIALS AND METHODS}

\section{Animals}

Approval was obtained for all experimental protocols from the University of Alabama at Birmingham Institutional Animal Care and Use Committee. All experiments were conducted in accordance with the Guide for the Care and Use of Laboratory Animals adopted by the National Institutes of Health. Young adult male Sprague-Dawley rats were housed in a $22 \pm 2^{\circ} \mathrm{C}$ room with food and water ad libitum. For a subset of experiments, young adult C57Bl/6 mice and adult Emx:ChR2 mice were used. The expression of ChR2 in excitatory neurons was accomplished

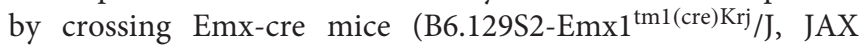
\#005628; Gorski et al., 2002) with floxed ChR2/EYFP mice Ai32 [RCL-ChR2 (H134R)/EYFP, JAX \#012569; Madisen et al., 2012]. $\mathrm{C} 57 \mathrm{Bl} / 6$ and Emx:ChR2 mice were housed in a $26 \pm 2{ }^{\circ} \mathrm{C}$ room with food and water ad libitum. Mice were housed with the whole litter until weaned (P25-P28). Weaned mice were housed with no more than seven mice in a cage. Mouse genotypes were determined from tail biopsies using real-time PCR with specific probes designed for cre and EYFP (Transnetyx, Cordova, TN, USA). Pregnant mice (CD-1) were group-housed (four mice to a cage) in a $22 \pm 2{ }^{\circ} \mathrm{C}$ room with food and water ad libitum and were monitored daily prior to use for neuronal cultures. The animal rooms maintained a standard 12-h light/dark cycle.

\section{Incubation of LSO:Ce Microparticles With Acute Hippocampal Slices}

Acute hippocampal slices were allowed to recover for an hour before application of either vehicle (artificial cerebral spinal fluid, aCSF) or $0.2-0.5 \mathrm{mg} / \mathrm{mL}$ of LSO:Ce radioluminescent microparticles. The slices were incubated with and without LSO:Ce microparticles for at least an hour, and up to $6 \mathrm{~h}$. Incubation of microparticles with hippocampal slices were performed using two types of chambers. Experiments measuring spontaneous postsynaptic currents and baseline synaptic transmission properties used a small incubation chamber that contained $5 \mathrm{mLs}$ of aCSF and was bubbled with $95 \% \mathrm{O}_{2}, \mathrm{pH} 7.35-7.45$ for the incubation period. For immunohistochemistry and LTP experiments, slices were placed in $2 \mathrm{mLs}$ of aCSF on top of a piece of filter paper within a humidified oxygenated interface recovery chamber, both for recovery and microparticle incubation.

\section{Electrophysiology Using Rats}

\section{Rat Hippocampal Slice Preparation}

Young adult male Sprague-Dawley rats (age 6-10 weeks; Charles River Laboratories) were anesthetized with isoflurane, decapitated, and brains removed; $400 \mu \mathrm{m}$ coronal slices from dorsal hippocampus were made on a VT1000P vibratome (Leica Biosystems) in oxygenated $\left(95 \% \mathrm{O}_{2} / 5 \% \mathrm{CO}_{2}\right)$ ice-cold high sucrose cutting solution (in $\mathrm{mm}$ as follows: $85.0 \mathrm{NaCl}$, $2.5 \mathrm{KCl}, 4.0 \mathrm{MgSO}_{4}, 0.5 \mathrm{CaCl}_{2}, 1.25 \mathrm{NaH}_{2} \mathrm{PO}_{4}, 25.0$ glucose, 75.0 sucrose). After cutting, slices were held at room temperature from 1 to $5 \mathrm{~h}$ with continuously oxygenated standard aCSF (in $\mathrm{mm}$ as follows: $119.0 \mathrm{NaCl}, 2.5 \mathrm{KCl}, 1.3 \mathrm{MgSO}_{4}, 2.5 \mathrm{CaCl}_{2}$, $1.0 \mathrm{NaH}_{2} \mathrm{PO}_{4}, 26.0 \mathrm{NaHCO}_{3}, 11.0$ glucose).

\section{Electrophysiology-Field Recordings}

Vehicle and LSO:Ce incubated slices were interleaved to account for slice health. Extracellular field excitatory postsynaptic potentials (fEPSPs) recorded from the dendritic region in hippocampal area CA1 were performed in a submersion chamber perfused with standard aCSF at room temperature. All data were obtained using the electrophysiology data acquisition software pClamp10 (Molecular Devices, LLC, Sunnyvale, CA, USA) and analyzed using Clampfit within the pClamp10 suite, and Graphpad Prism 7 (GraphPad Software, Inc.). For CA3-CA1 synapses, Schaffer collateral axons were stimulated using a twisted insulated nichrome wire electrode placed in CA1 stratum radiatum within 200-300 $\mu \mathrm{m}$ of an aCSF-filled glass recording electrode, and paired-pulse facilitation (PPF) characteristic of this synapse (Wu and Saggau, 1994) was recorded. Baseline fEPSPs were obtained by delivering $0.1 \mathrm{~Hz}$ stimulation for $200 \mu \mathrm{s}$ to generate fEPSPs of $0.2-0.3 \mathrm{mV}$ in amplitude. Only experiments with $\leq 10 \%$ baseline variance were included in the final data sets.

\section{Input-Output Curves}

After a stable $10 \mathrm{~min}$ baseline, input-output (I/O) curves were generated by increasing the stimulus intensity $(20 \mu \mathrm{A}$ increments) until a maximal fEPSP slope was obtained, usually at $200 \mu \mathrm{A}$. Initial slope of the five fEPSPs generated at each stimulus intensity was averaged and plotted as a single value. Statistical significance was determined by two-way ANOVA with Sidak's multiple comparison test.

\section{Paired Pulse Ratio}

After a 10 min stable baseline, pairs of stimulation were delivered at a 50 millisecond (ms) inter-stimulus interval (ISIs). The paired pulse ratio (PPR) was calculated by dividing the initial slope of the second fEPSP by the initial slope of the first fEPSP.

\section{Long Term Potentiation}

At CA3-CA1 synapses, following a $10 \mathrm{~min}$ stable baseline $(0.1 \mathrm{~Hz}, 200 \mu \mathrm{s}$ with stimulation intensity set to elicit initial fEPSP amplitude of 0.3-0.4 mV), NMDA receptor (NMDAR)dependent LTP was induced using high-frequency stimulation (HFS, $100 \mathrm{~Hz}, 1 \mathrm{~s}$ duration $\times 5,60 \mathrm{~s}$ interval). Statistical significance was determined using unpaired Student's $t$-test by comparing the average of the fEPSP slope from the last $5 \mathrm{~min}$ of the recording $(35-40 \mathrm{~min})$ to baseline for each genotype $\left({ }^{*} p<0.05\right)$.

\section{Electrophysiology -Whole Cell}

All recordings were performed in a submersion chamber with continuous perfusion of oxygenated standard aCSF. Whole-cell voltage clamp recordings were carried out in blind patched CA1 pyramidal neurons. Inhibitory postsynaptic currents (IPSCs) were pharmacologically isolated with bath perfusion of DNQX (10 $\mu \mathrm{M}$; Sigma) and DL-AP5 (50 $\mu \mathrm{M}$; Tocris). Spontaneous IPSCs (sIPSCs) were recorded using $\mathrm{CsCl}$ internal solution (in mM: $140.0 \mathrm{CsCl}, 10.0$ EGTA, $5.0 \mathrm{MgCl}_{2}, 2.0 \mathrm{Na}-\mathrm{ATP}$, 
$0.3 \mathrm{Na}-\mathrm{GTP}, 10.0$ HEPES; $\mathrm{E}_{\mathrm{Cl}}=0 \mathrm{mV}$ ). All cells were dialized for 3-7 min prior to the beginning of experimental recordings. Stability of series resistance during the recording was verified posthoc through comparing the average rise and decay time of sIPSCs. Recordings were discarded if the rise or decay time changed by $>20 \%$.

\section{Electrophysiology in Mice \\ Mouse Hippocampal Slice Preparation}

Male and female mice, 4-7 months of age, were anesthetized with isoflurane and sacrificed by decapitation using a rodent guillotine. Due to the age of the mice, a more neuroprotective dissection solution was used during acute hippocampal slice preparation (Tanaka et al., 2008). The brains were rapidly removed and placed in ice-cold dissection solution containing the following (in $\mathrm{mM}$ ): $135 \mathrm{~N}$-Methyl-D-glucamine, $1.5 \mathrm{KCl}$, $1.5 \mathrm{KH}_{2} \mathrm{PO}_{4}, 0.5 \mathrm{CaCl}_{2}, 3.5 \mathrm{MgCl}_{2}, 23 \mathrm{NaHCO}_{3}, 0.4 \mathrm{~L}$-Ascorbic acid, and 10 glucose, bubbled with 95\% $\mathrm{O}_{2} / 5 \% \mathrm{CO}_{2}, \mathrm{pH}$ 7.35-7.45, and osmolarity 295-305 (Albertson et al., 2017). A vibratome (Campden $7000 \mathrm{smz}-2$, Lafayette Instrument) was used to cut $300 \mu \mathrm{M}$ thick hippocampal brain slices. The slices were maintained for $45-60 \mathrm{~min}$ at $37^{\circ} \mathrm{C}$ in oxygenated recovery solution containing (in $\mathrm{mM}$ ) $120 \mathrm{NaCl}, 3.5 \mathrm{KCl}, 0.7 \mathrm{CaCl}_{2}$, $4.0 \mathrm{MgCl}_{2}, 1.25 \mathrm{NaH}_{2} \mathrm{PO}_{4}, 26 \mathrm{NaHCO}_{3}$, and 10 glucose and then kept at room temperature. Slices were stored at room temperature in a holding chamber containing the recovery solution and bubbled with $95 \% \mathrm{O}_{2} / 5 \% \mathrm{CO}_{2}$ for $>30 \mathrm{~min}$ before recording.

\section{Whole Cell Electrophysiology Recording}

Photostimulation of the microparticles occurred by using pulses of ultraviolet (UV) light $(315 \mathrm{~nm}$ or $365 \mathrm{~nm}, 500 \mathrm{~ms}$ pulse duration). The $365 \mathrm{~nm}$ UV light was generated by a Colibri.2 LED Light Source (Zeiss) applied to acute hippocampal slices through a $10 \times$ objective, resulting in an illumination area of $5.29 \mathrm{~mm}^{2}$. The $365 \mathrm{~nm}$ light intensity was measured as being approximately $0.003 \mathrm{~mW} / \mathrm{mm}^{2}$ by ThorLabs PM100D Optical Power Meter. The $315 \mathrm{~nm}$ UV light was generated by custom building the unit using components obtained from ThorLabs (Figure 1). The $315 \mathrm{~nm}$ UV light is applied to the acute hippocampal slice through a $2.5 \mathrm{~mm}$ stainless steel ferrule, therefore allowing a similar illumination area to that of the $10 \times$ objective. Both the Colibri. 2 and the custom-built $315 \mathrm{~nm}$ portable unit were triggered by a Master- 9 digital stimulator (A.M.P.I).

For the recordings, slices were placed in a submersion recording chamber and perfused (3-4 $\mathrm{ml} / \mathrm{min}$ ) with aCSF. Experiments were performed at $28-32^{\circ} \mathrm{C}$. For spontaneous EPSC (sEPSC) recordings, CA1 pyramidal cells were blindly patched on a Zeiss Examiner A1 upright microscope. Neurons were patched in the voltage-clamp configuration and recorded at a holding potential of $-60 \mathrm{mV}$ using an Axopatch 200B amplifier (Molecular Devices). Patch electrodes (4-6 M $\Omega$ ) were filled with a potassium gluconate based internal solution composed of the following (in $\mathrm{mM}$ ): $130 \mathrm{~K}$-gluconate, $0.1 \mathrm{EGTA}, 20 \mathrm{KCl}$, $2 \mathrm{MgSO}_{4} \cdot 7 \mathrm{H}_{2} \mathrm{O}, 10 \mathrm{HEPES}, 5$ phosphocreatine-tris, 10 ATP, and 0.3 GTP. The $\mathrm{pH}$ was adjusted to 7.2 with $\mathrm{KOH}$ and osmolarity was 290-295. The access resistance and holding current $(<200$ pA) were monitored continuously. Recordings were rejected if either access resistance or holding current increased $\geq 25 \%$ during the experiment. The access resistance for experiments performed without the particles was $29.6 \pm 2.4 \mathrm{M} \Omega$ and the slices with LSO:Ce microparticles was $26.2 \pm 1.9 \mathrm{M} \Omega$ ( $n=16,12$ cells respectively, unpaired $t$-test, $p>0.3$ ).

Analysis of sEPSC frequency and amplitude were performed using custom software written in Visual Basic, which measured amplitude and interevent interval. Events were fit to a template response and all events that fit the template and passed visual inspection were included in the analysis. The rise time and the decay time constant were measured from averaged sEPSC events for each individual experiment. The rise time is defined as the time required to travel from $20 \%$ to $80 \%$ of the amplitude. The decay time constant was determined by fitting with a single exponential function equation.

\section{Immunohistochemistry}

Acute $300 \mu \mathrm{m}$ hippocampal slices were obtained from P50 to P70 C57Bl/6 mice. Slices were incubated with and without $0.25-0.5 \mathrm{mg} / \mathrm{mL}$ of LSO:Ce RPLs for $0,1,3$, and $6 \mathrm{~h}$. Slices were fixed overnight at $4^{\circ} \mathrm{C}$ in $4 \%$ paraformaldehyde in $0.1 \mathrm{M}$ phosphate buffered saline (PBS) and immunohistochemistry was performed based on a modified protocol (Dissing-Olesen and MacVicar, 2015; Miller et al., 2017; Yang et al., 2018). Briefly, the free-floating fixed slices were washed five times for $10 \mathrm{~min}$ in PBS. The slices were permeabilized for $1 \mathrm{~h}$ at room temperature in $0.1 \mathrm{M}$ PBS (pH 7.4) with $0.3 \%$ Triton X-100 and $20 \%$ DMSO (PBSTD). Slices were blocked with $10 \%$ donkey serum (cat\# 017-000-121, Jackson ImmunoResearch Inc.) in PBSTD for $3 \mathrm{~h}$ at room temperature. Following blocking, the sections were immunostained with a monoclonal mouse antibody against glial fibrillary acidic protein (GFAP; 1:1,000, cat\# 3670, Cell Signaling Technology) for $48-96 \mathrm{~h}$ at $4^{\circ} \mathrm{C}$ in blocking solution. After washing, the slices underwent another blocking step. Then the slices were incubated with Alexa Fluor 488 conjugated polyclonal donkey anti-mouse antibody secondary (cat\# 715-545-150, Jackson ImmunoResearch Inc.) diluted 1:400 in blocking solution for $16-40 \mathrm{~h}$ at $4^{\circ} \mathrm{C}$. Staining specificity was confirmed by the omission of primary antibody. Sections were mounted with the nuclear dye 4',6-diamindino-2-phenylindole dihydrochloride (DAPI) in the VECTASHIELD HardSet mounting medium (cat\# H-1500, Vector Laboratories). Immunostained slices were imaged using an epifluorescent Nikon eclipse ni microscope and NIS Elements v. 4.20.02 software. To individually count CA1 pyramidal cells, images were taken using the $40 \times$ objective of the DAPI stain. Images for GFAP analysis were taken using a $10 \times$ objective. Analysis of images was performed using ImageJ (Fiji).

\section{Primary Cortical Neuronal Culture}

Mouse primary cortical neuronal culture from pregnant CD-1 mice was made as previously described (Andrabi et al., 2014). Embryos obtained from mice at Day 15-16 of gestation were used to prepare primary cortical neuron cultures. Briefly, the cortical regions of the embryonic brains were aseptically 
dissected, freed of meninges and dissociated in dissecting medium (DMEM + 20\% fetal bovine serum) and subjected to trypsin digestion at $37^{\circ} \mathrm{C}$ for $5 \mathrm{~min}$. Tryptic digestion was stopped by the addition of dissecting medium and the cell suspension was centrifuged at $1,000 \mathrm{~g}$ for $5 \mathrm{~min}$. Next, the pelleted cells were subjected to mechanical trituration in complete Neurobasal medium (10 mM glucose, $1 \mathrm{mM}$ GlutaMAX-I, $1 \mathrm{mM}$ Na-Pyruvate and 2\% B-27) and passed through a $40 \mu \mathrm{m}$ filter. The cells were plated to a density of $5 \times 10^{5}$ cells $/ \mathrm{ml}$. On day in vitro (DIV) 1 , the cultures were treated with 5 -fluoro 2 -deoxyuridine $(40 \mu \mathrm{M})$ to inhibit glial cell growth and proliferation. Experiments were performed between DIV 10 and 11. Under these conditions, mature neurons represent $90 \%$ of the cells in the culture.

\section{Cell Death Assay}

Neurons at DIV 10 were incubated with either microparticles (LSO:Ce, dispersed in PBS), at concentrations ranging from 0.05 to $0.2 \mathrm{mg} / \mathrm{ml}$, or PBS for $24 \mathrm{~h}$. Cell viability after addition of nanoparticles was determined by using Alamar blue reagent, a water-soluble resazurin dye (blue colored) which is reduced to red fluorescent resorufin dye by metabolically active cells. Alamar blue reagent $[10 \%(\mathrm{v} / \mathrm{v})]$ was then added to each well containing Neurobasal medium and incubated for $3 \mathrm{~h}$ at $37^{\circ} \mathrm{C}$. Blank control well containing microparticles only were used to exclude possible interactions with the assay. Neurobasal medium containing Alamar blue reagent was added to these wells. Following $3 \mathrm{~h}$ of incubation, $100 \mu \mathrm{L}$ of the medium was collected from each well and transferred to a 96-well microplate. The fluorescence was measured at the excitation and emission wavelength of 540 and $595 \mathrm{~nm}$, respectively using a microplate reader. The fluorescence values were normalized by the control (PBS) and expressed as percent viability.

\section{Materials}

Commercial lutetium oxyorthosilicate particles (median particle size: $4 \mu \mathrm{m}$ ) were purchased from Phosphor Technologies and were doped with cerium at a 1-10 atomic \% cerium (LSO:Ce). Prior to their use, the particles were washed with deionized water and vacuum air-dried.

\section{RESULTS}

\section{Light Emitted From LSO:Ce Particles Can Weakly Activate Channelrhodopsin-2}

LSO:Ce particles emit within the activation spectrum for ChR2. Interestingly, these particles can be activated with UV light. As a proof of principle, we took advantage of this property to test whether light emitted from LSO:Ce microparticles could enhance synaptic activity. However, it was necessary to first test whether ChR2 is activated by UV light. We used Emx:ChR2 mice to determine the extent to which ChR2 is activated by various wavelengths of UV light. Using UV light at $365 \mathrm{~nm}$, we observed a photocurrent $(-22.4 \pm-2.3 \mathrm{pA}, n=10$ cells $)$ in recordings from CA1 pyramidal cells in acute slices, even with a relatively low light intensity $\left(0.003 \mathrm{~mW} / \mathrm{mm}^{2}\right)$. We were concerned that the activation of ChR2 with $365 \mathrm{~nm}$ light would hamper our ability to see the activation of ChR2 from light generated by the LSO:Ce particles. Therefore, we built a portable UV system that would emit light around $315 \mathrm{~nm}$ (Figure 1). Application of $315 \mathrm{~nm}$ light onto CA1 pyramidal cells that express ChR2 generated an extremely small, but detectable, photocurrent $(-5.5 \pm-2.0 \mathrm{pA}$, $n=5$ cells, Figure 2 B).

As the light emitted from the LSO:Ce particles might not be uniform enough to generate a synchronized synaptic event, we analyzed the impact on the amplitude and frequency of

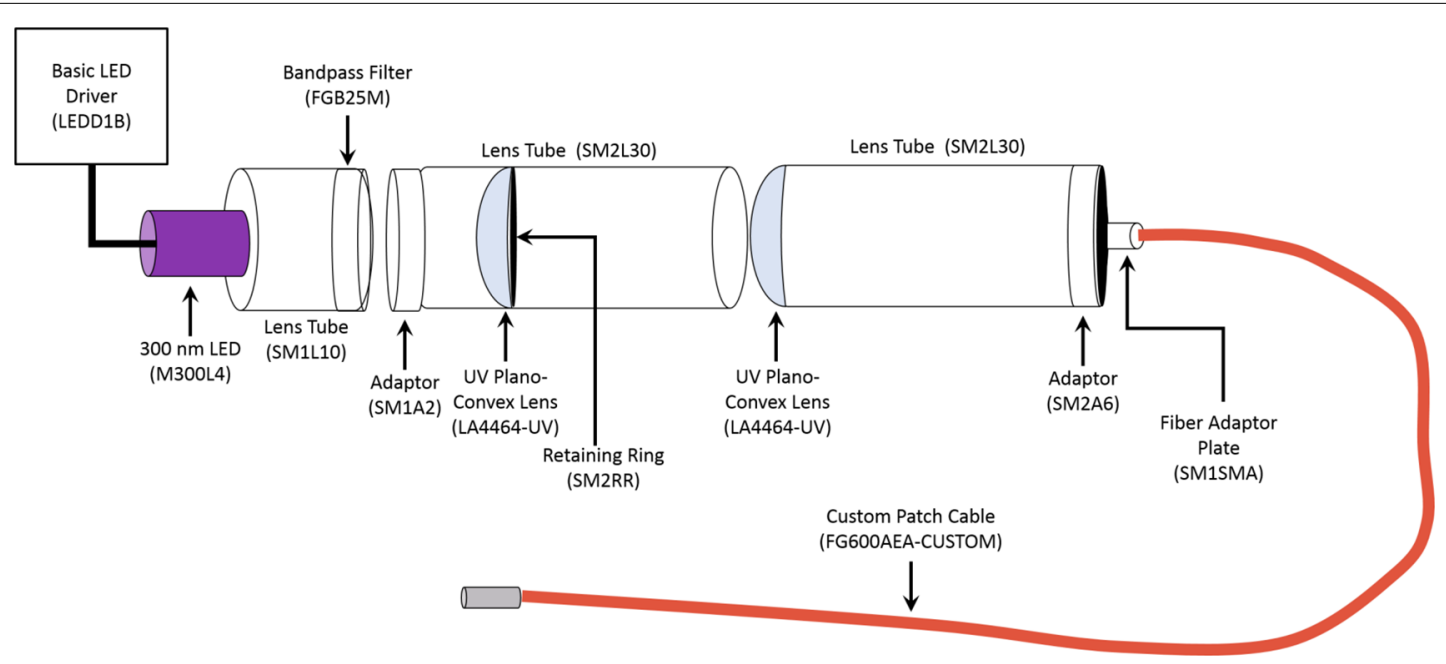

FIGURE 1 | Schematic of Portable $315 \mathrm{~nm}$ ultraviolet (UV) light emitting diode (LED). All the components were obtained from ThorLabs. The general name of each component is labeled, and the part number is in the parentheses. The LED Driver is the power source for the unit. The emission spectrum for the 300 nm LED ranges from approximately $285 \mathrm{~nm}$ to $325 \mathrm{~nm}$, with it peaking at $300 \mathrm{~nm}$. The bandpass filter allows transmission between $315 \mathrm{~nm}$ to $445 \mathrm{~nm}$, thereby narrowing the range of emitted light to $315-320 \mathrm{~nm}$. The custom $1 \mathrm{~m}$ long patch cable had the following parameters: Fiber-FG600AEA, Tubing-FT038, End 1-SMA, and End 2-2.5 mm (FC) stainless steel ferrule. 

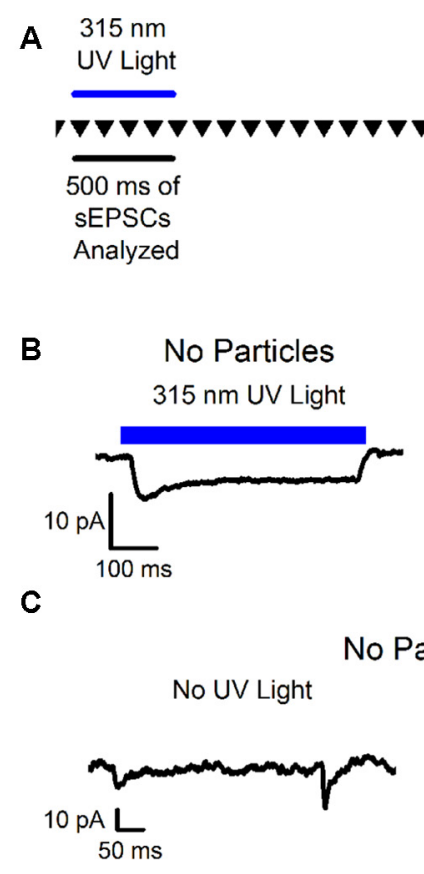

No Particles

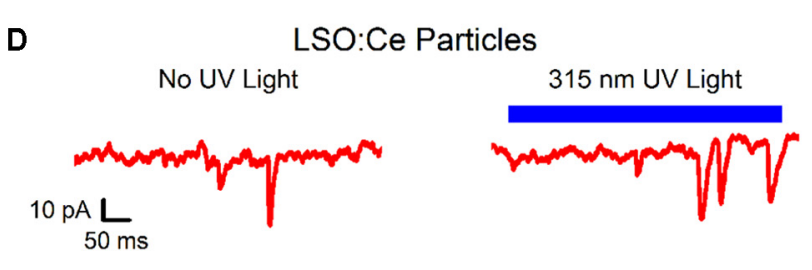

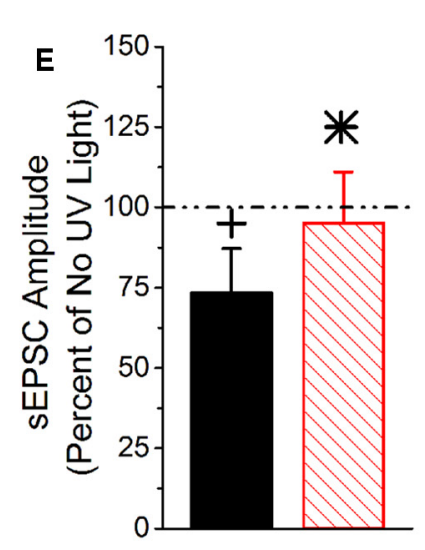

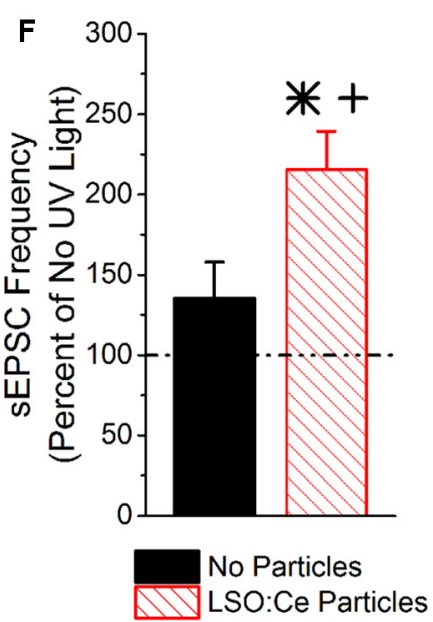

FIGURE 2 | Light emitted from Cerium doped lutetium oxyorthosilicate (LSO:Ce) microparticles enhances synaptic transmission from channelrhodopsin-2 (ChR2) expressing CA1 pyramidal cells. (A) Experimental schematic that shows the interleave nature of the application of the $315 \mathrm{~nm}$ UV light and the analysis. Every $10 \mathrm{~s}$, traces that lasted for $3 \mathrm{~s}$ were recorded (depicted by the inverted triangles), and each trace was repeated 50-100 times. The spontaneous excitatory postsynaptic currents (sEPSC) events for each individual trace was analyzed during the $500 \mathrm{~ms}$ when the UV light was on or at the end of the trace when there was no UV light. (B) Example averaged trace (at least 25 traces) of $315 \mathrm{~nm}$ induced photocurrents in acute hippocampal Emx:ChR2 mouse slices with (red trace) or without (black trace) LSO:Ce particles. (C) Example of an individual trace of sEPSCs recorded from a CA1 pyramidal cell in an acute hippocampal slice from an Emx:ChR2 mouse that was not incubated with LSO:Ce particles in the absence (left) or presence (right) of the $315 \mathrm{~nm}$ UV light. (D) Example of an individual trace of sEPSCs onto CA1 pyramidal cells from an acute hippocampal Emx:ChR2 mouse slice that was incubated with LSO:Ce particles for at least $1 \mathrm{~h}$ in the absence (left) or presence (right) of the $315 \mathrm{~nm}$ UV light. (E,F) For each experiment, the sEPSC amplitude and frequency measured in the presence of the $315 \mathrm{~nm}$ UV light was normalized to a section of the same trace that was not exposed to UV light to account for cell to cell variability and recording stability. (E) Application of UV light alone decreased the amplitude of sEPSCs compared to baseline ( $n=5$ cells $/ 4$ animals, Student's $t$-test, $p=0.01)$. Nevertheless, the presence of the LSO:Ce particles enhanced the sEPSC amplitude as compared to UV alone ( $n=5$ cells/4 animals no particles, 7 cells $/ 5$ animals LSO:Ce particles, Student's $t$-test, $p=0.03$ ). (F) There was a trend for exposure to UV light alone to enhance the frequency of sEPSCs ( $n=5$ cells $/ 4$ animals, Student's $t$-test, $p=0.19$ ). The light emitted from LSO:Ce particles by UV activation almost doubles the number of sEPSCs compared to baseline ( $n=5$ cells/4 animals no particles, 7 cells $/ 5$ animals LSO:Ce particles, Student's $t$-test, $p=0.04) .{ }^{*}$ Indicates significant difference $(p<0.05)$ with and without LSO:Ce particles; ${ }^{+}$Indicates significant difference $(p<0.05)$ compared to no UV light.

sEPSCs. As there is cell to cell variability with sEPSCs, we used a within cell control (no UV light) to normalize the amplitude and frequency of sEPSCs. Figure 2A depicts the experimental paradigm showing that the periods of UV light stimulation and no stimulation were interleaved throughout the experiment. Even though only a small photocurrent was generated with the application of $315 \mathrm{~nm}$ light, there was a trend for an increase in the frequency of sEPSCs during UV light exposure (Figures 2C,F). Importantly, the enhancement is much larger when the LSO:Ce particles are present (Figures 2D,F). A similar enhancement of the frequency of sEPSCs was seen when $365 \mathrm{~nm}$ UV light was applied (UV only $149.4 \pm 20.9 \%$ vs. UV + LSO:Ce $225.2 \pm 24.4 \%, n=8$ cells, 7 cells, Student's $t$-test, $p=0.04$ ). Interestingly, the amplitude of the sEPSCs is decreased with the application of $315 \mathrm{~nm}$ light (Figure 2E). However, the addition of the LSO:Ce particles enhances the amplitude of the sEPSCs compared to UV alone (Figure 2E). Additionally, the overall size of the photocurrent induced by the $315 \mathrm{~nm}$ UV light was enhanced in the presence of the particles as compared to UV light application alone (Vehicle slices $-5.5 \pm-2.0 \mathrm{pA}$, vs. LSO:Ce 
slices $-12.3 \pm 1.8 \mathrm{pA}, n=5$ cells $/ 4$ animals, 7 cells $/ 5$ animals, Student's $t$-test, $p=0.03$, Figure 2B). These data indicate that the light emitted from LSO:Ce particles is able to activate ChR2 and increase the frequency of sEPSCs.

\section{Prolonged Exposure to LSO:Ce Microparticles Does Not Alter Neuronal Survival or Properties}

We next tested the biocompatibility of LSO:Ce microparticles. Neurons are extremely sensitive to changes in their environment and any perturbations could lead to neuronal cell death, in as little as $4 \mathrm{~h}$ (Hoque et al., 2019). We determined if prolonged exposure to LSO:Ce particles had any effect on neuronal survival using the Alamar Blue assay and primary cortical cultures. The result demonstrates no significant sign of toxicity exerted by the microparticles at any of the doses tested, even with $24 \mathrm{~h}$ of exposure to the LSO:Ce microparticles (Figure 3).

Primary cortical cultures are more sensitive to extracellular perturbations than acute slices. However, we confirmed the results that neuronal survival was not affected by acute application of the LSO:Ce microparticles using acute hippocampal slices. We incubated slices with $0.25-0.5 \mathrm{mg} / \mathrm{mL}$ of LSO:Ce microparticles for $0-6 \mathrm{~h}$, and manually counted the number of DAPI positive neurons in the pyramidal layer. We saw no difference in the density of CA1 pyramidal cells between unexposed and exposed slices even after $6 \mathrm{~h}$ of treatment (No particle slices $109.0 \pm 8.9 \mathrm{cells} / \mathrm{mm}^{2}$ vs. LSO:Ce slices $105.9 \pm 16.3$ cells $/ \mathrm{mm}^{2}, n=11$ images $/ 4$ animals, 9 images $/ 4$ animals, Student's $t$-test, $p=0.86$ ). Additionally, acute incubation of LSO:Ce particles did not change the resting membrane potential of CA1 pyramidal cells (No particle slices $-63.9 \pm 2.7 \mathrm{mV}$ vs. LSO:Ce particle slices $-66.1 \pm 2.1 \mathrm{mV}, n=16$ cells/11 animals, 13 cells $/ 7$ animals, Student's $t$-test, $p=0.55$ ). However, the input resistance of the cells was slightly reduced (No particle slices $99.1 \pm 6.6 \mathrm{M} \Omega$ vs. LSO:Ce particle slices $80.4 \pm 5.1 \mathrm{M} \Omega$, $n=16$ cells $/ 11$ animals, 13 cells $/ 7$ animals, Student's $t$-test, $p=0.04)$. Overall, exposure to LSO:Ce microparticles did not affect neuronal health.

Implanted probes have been shown to increase glial scarring (Canales et al., 2018), which takes several days to develop (Wanner et al., 2013). One of the early steps in glial scar development is the activation of astrocytes. GFAP can be used as a way to measure astrocyte activation and has been shown to be increased within $2-4 \mathrm{~h}$ after damage (Schmidt-Kastner et al., 1993). Therefore, to determine if astrocytes became reactive in the presence of these particles, we used immunohistochemistry to analyze GFAP in LSO:Ce preincubated slices compared to control slices (no particles). As the LSO:Ce particles could potentially influence the visualization of GFAP staining, we performed control experiments in which we immediately processed slices after application of vehicle (aCSF) or the particles (Figure 4) to test for differences in GFAP fluorescent intensity caused by light being emitted or absorbed by the particles. However, the overall fluorescent intensity of GFAP staining was not different between vehicle-treated slices

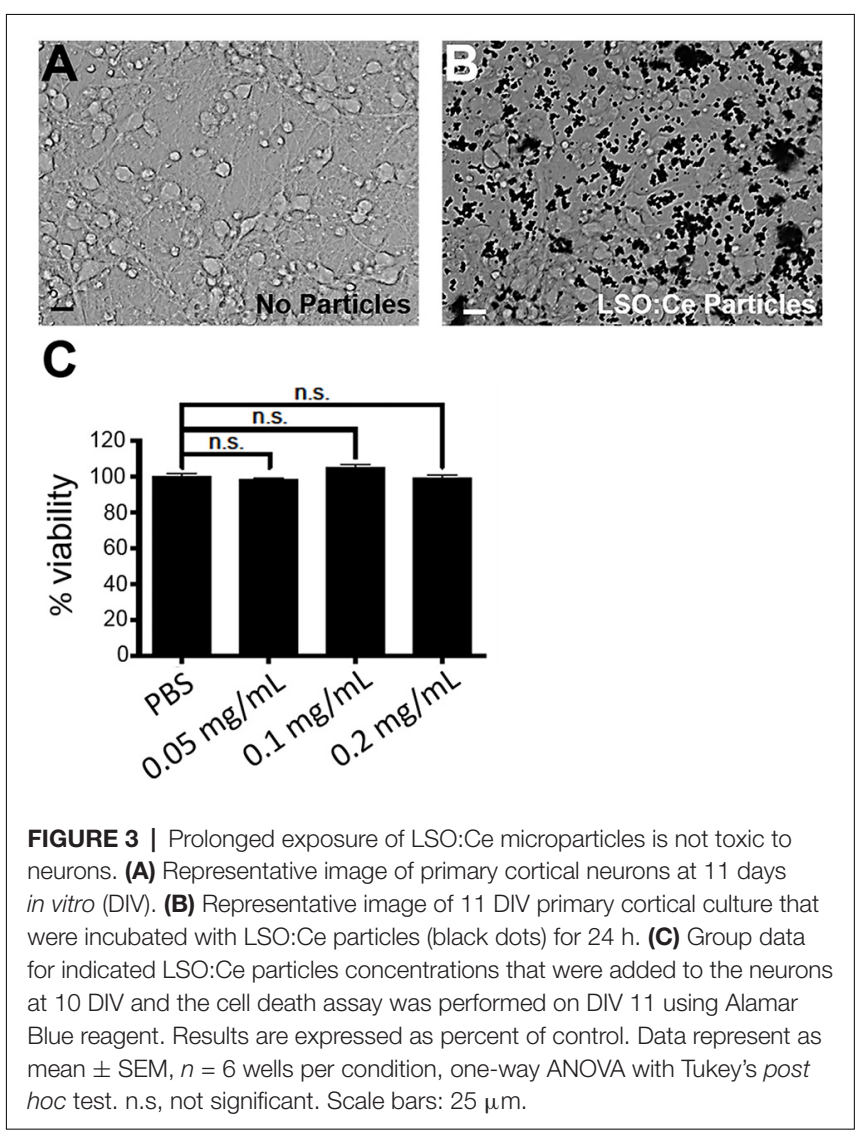

and LSO:Ce-treated slices at the $0 \mathrm{~h}$ time point, indicating that measurement of GFAP fluorescence was not altered due to the emission and absorbance capabilities of the LSO:Ce particles (No particles slices $1.03 \times 10^{6} \pm 1.14 \times 10^{5} \mathrm{vs}$. LSO:Ce particles slices $1.02 \times 10^{6} \pm 6.02 \times 10^{4}, n=8$ slices $/ 4$ animals, 8 slices $/ 4$ animals, Student's $t$-test, $p=0.98$ ). Since the LSO:Ce particles fluorescent properties do not alter our ability to visualize GFAP staining, we can determine if their presence over time causes changes in the GFAP intensity. Slices were incubated for up to $6 \mathrm{~h}$ with either vehicle or the LSO:Ce microparticles. The results show that there was no increase in the level of GFAP staining due to prolonged incubation with the LSO:Ce particles (Figure 4). Our data suggest that there is no gross changes in astrocyte activation with the application of LSO:Ce particles.

\section{LSO:Ce Microparticles Modestly Alter Synaptic Transmission}

Even though overall cell health was unaltered by exposure to LSO:Ce particles, the synaptic transmission could still be affected. We further analyzed this by incubating hippocampal slices with LSO:Ce particles for 1-3 h, followed by recording extracellular dendritic fields potentials (fEPSPS) from CA1 pyramidal cells in stratum radiatum in response to increasing the number of CA3 Schaffer collateral axons stimulated (Figures $\mathbf{5 A - C}$ ). The initial slope of the fEPSP represents the postsynaptic response, and we found no difference in the maximal response generated between the control and LSO:Ce-treated slices (Figure 5B). 

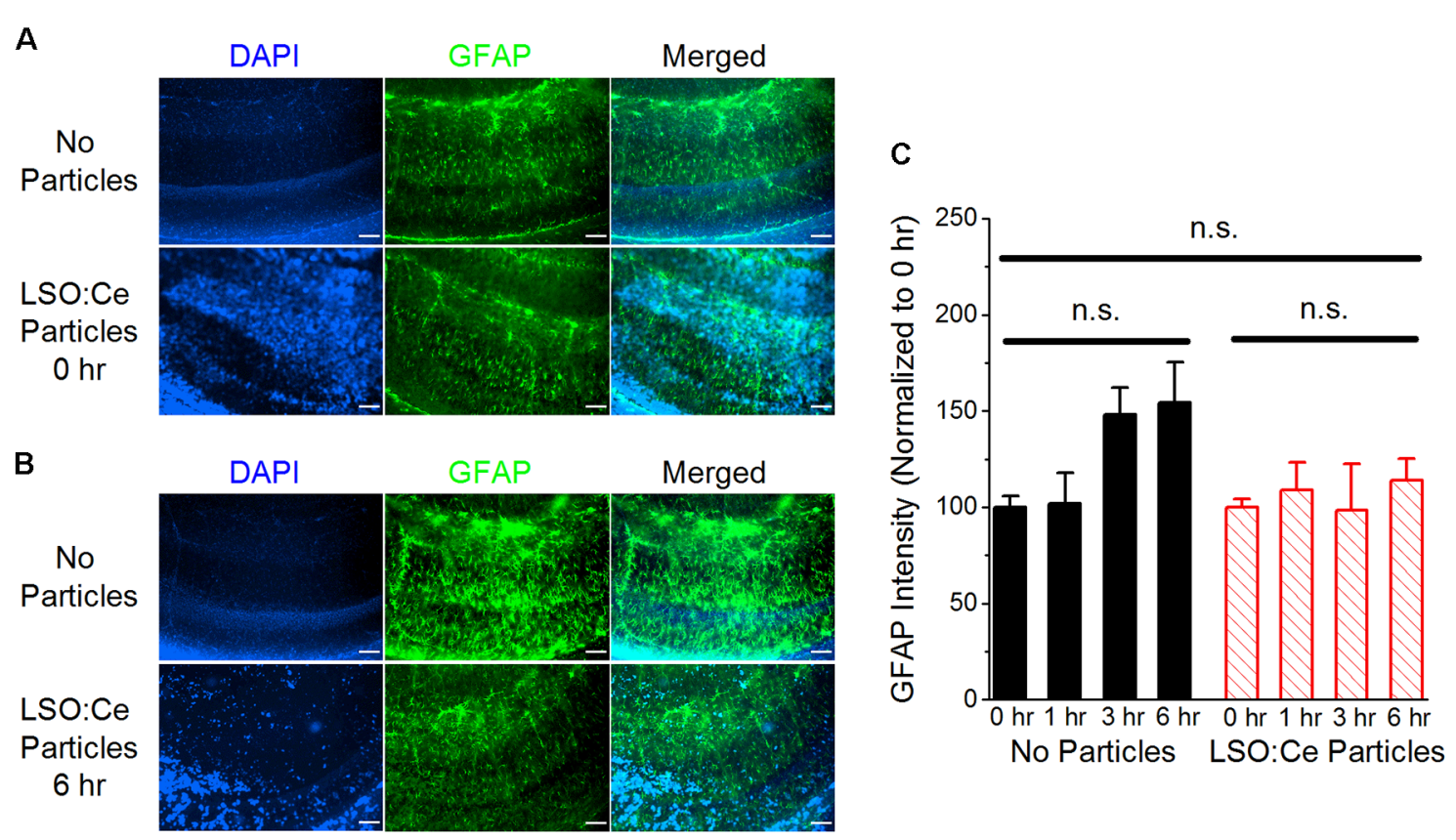

FIGURE 4 | Exposure of LSO:Ce microparticles to acute hippocampal slices does not elevate glial fibrillary acidic protein (GFAP). (A) Example images of GFAP staining in acute hippocampal slices immediately after application of vehicle (no particles) or LSO:Ce particles. Merged images include 4',6-diamindino-2-phenylindole dihydrochloride (DAP; blue) and GFAP (green) staining. Scale bars: $100 \mu \mathrm{m}$. (B) Example images of GFAP staining in acute hippocampal slices $6 \mathrm{~h}$ after application of vehicle or LSO:Ce particles. Merged images include DAPI (blue) and GFAP (green) staining. Scale bars: $100 \mu \mathrm{m}$. (C) Group data shows that the intensity of GFAP staining is not altered with the addition of the LSO:Ce particles $\left(n=8\right.$ slices $/ 4$ animals per condition, two-way ANOVA, $\left.F_{(3,63)}=1.76, p=0.17\right)$. n.S, not significant.

However, a small decrease in the amplitude of the fiber volley, a measurement of the number of axons activated, was seen with high stimulation (Figure 5C). In addition, we saw a small, but significant, increase in the PPR, suggesting that the LSO:Ce particles may alter presynaptic function (Figure 5D).

To further investigate whether incubation with LSO:Ce particles affects synaptic transmission, we preincubated acute hippocampal slices with LSO:Ce particles for 1-3 h. We then performed whole-cell recordings from CA1 pyramidal cells and recorded spontaneous excitatory (sEPSCs; Figure 6) and inhibitory postsynaptic currents (sIPSCs; Figure 7). There was no change in the amplitude of sEPSCs (Figures 6A,B), however, the frequency of the events was slightly but significantly reduced with exposure to LSO:Ce particles (Figures 6A,C). On the other hand, sIPSCs frequency and amplitude were unaltered in the presence of the particles (Figure 7). Additionally, the kinetics of the sEPSCs (Figures 6D,E) and sIPSCs (Figure 7C) were unaltered in the presence of the LSO:Ce microparticles. This suggests either that the particles are reducing the number of excitatory synapses, modifying presynaptic function, or altering axonal excitability.

\section{LSO:Ce Microparticles Modestly Alter Long Term Potentiation}

Next, we asked if long-term plasticity, a more robust measure of synaptic health and integrity, is compromised by pre-incubation with LSO:Ce particles. LTP, an increase in synaptic strength that lasts for at least $40 \mathrm{~min}$ in vitro and hours to days in vivo and is thought to underlie learning and memory, has been extensively characterized at CA3-CA1 synapses, and involves the insertion of excitatory AMPA receptors (AMPARs) on post-synaptic CA1 pyramidal cells. We again incubated acute hippocampal slices for 1-3 h with LSO:Ce particles, and then recorded extracellular fEPSPs in response to Schaffer collateral stimulation in an interleaved fashion where control and LSO:Ce microparticles treated slices were alternated. Following the establishment of a 10-min baseline, we electrically induced LTP at CA3-CA1 synapses by stimulating Schaffer collateral axons at $100 \mathrm{~Hz}$ for $0.5 \mathrm{~s}$, five times, separated by intervals of $20 \mathrm{~s}$. While this protocol produced identical post-tetanic potentiation and LTP up to 20 min post-tetanus in control and LSO:Ce treated slices, there was a very small but significant decrease in LTP expression at 40 min post-tetanus in the LSO:Ce treated vs. control slices, which may suggest that the particles have a slightly negative impact on long-term expression of LTP (Figure 8).

\section{DISCUSSION}

Our results provide proof of principle that light from LSO:Ce microparticles can activate ChR2 and modulate synaptic function. In addition, we demonstrate that LSO:Ce particles are not overtly toxic to neurons, as there was no effect of the particles on neuronal survival in culture even with incubations as long as $24 \mathrm{~h}$. Synaptic function and plasticity also appear to be intact, as 


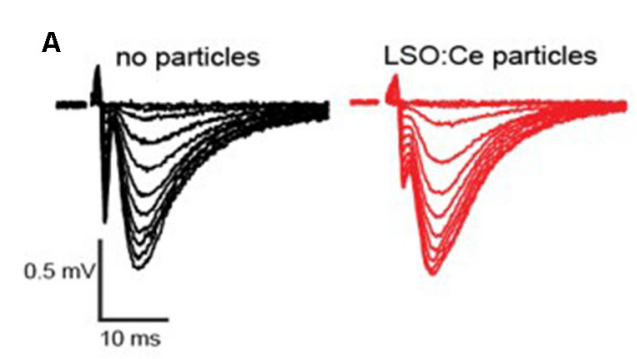

C

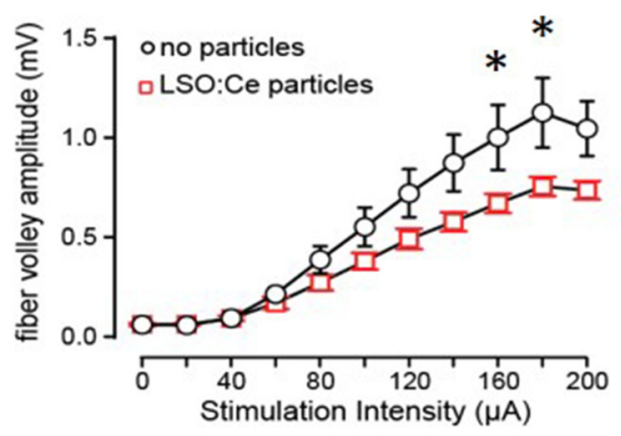

$\mathbf{B}$

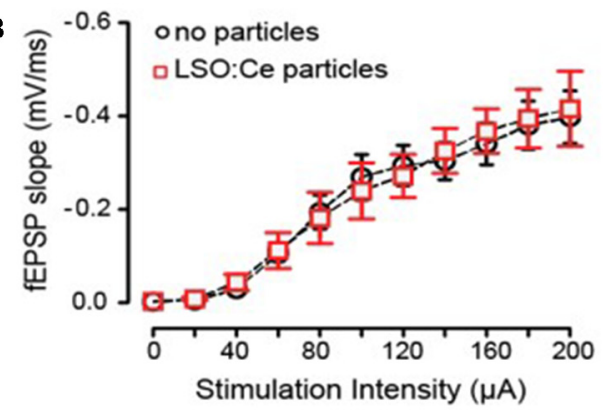

D

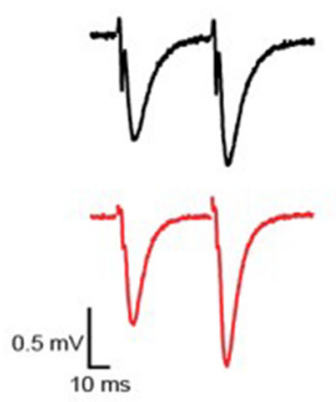



FIGURE 5 | Acute application of LSO:Ce microparticles has no effect on the strength of basal synaptic transmission but reduces axonal excitability at CA3-CA1 synapses. (A) Representative traces of field excitatory postsynaptic potentials (fEPSPs) at CA3-CA1 synapses in response to increasing stimulus intensity from acute hippocampal slices incubated without and with LSO:Ce particles (black traces: no particles and red traces: particles). (B) No change in the input/output (I/O) curve in in the presence of LSO:Ce particles ( $n=6$ slices/6 animals no particles, $n=6$ slices/6 animals LSO:Ce; ${ }^{*} p>0.05$ ). Data represent mean \pm SEM. Significance determined by two-way ANOVA with Sidak's multiple comparison test. (C) Group data showing a reduction in fiber volley amplitude with increasing stimulus intensity in the presence of LSO:Ce particles ( $n=6$ slices $/ 6$ animals no particles, $n=6$ slices $/ 6$ animals LSO:Ce; two-way ANOVA, $F_{(1,110)}=24.48, p<0.001$, ${ }^{*} p<0.05$ with Sidak's multiple comparisons test. (D; left) Representative traces of paired fEPSPs (black traces: no particles and red traces: particles). (right) Paired-pulse ratio (PPR) was significantly increased following incubation with particles ( $n=6$ slices/6 animals control, $n=6$ slices/6 animals LSO:Ce; * $p<0.05$ ).

there was no change in the input/output relationship in CA1, and LTP was able to be induced. However, there were indications that LSO:Ce particles themselves have effects on synaptic function and plasticity, although the effects were small. Together, these results suggest that LSO:Ce particles could potentially be suitable for in vivo optogenetics.

LSO:Ce was introduced as a scintillator in the early 90s (Melcher and Schweitzer, 1992) and has been extensively characterized since. Cerium forms the luminescence centers in LSO, and the Ce doped LSO displays a high emission intensity under X-ray illumination, with an emission maximum at $420 \mathrm{~nm}$. This emission peak makes LSO:Ce well suited for optogenetics applications using ChR2, which has maximal activation using $450 \mathrm{~nm}$ light (Berndt et al., 2011; Lin, 2011). The use of X-rays has the distinct advantage over implanted LEDs to activate ChR2, as $\mathrm{X}$-rays are able to penetrate the skull, removing the need for an invasive delivery method. However, before this study, it was not known if LSO:Ce particles themselves could emit enough light to activate ChR2.

Implantation of LEDs or a fiber requires the light output to be proportionally higher than necessary to activate ChR2 because of light scattering properties in brain tissue. One study using a fiber to deliver light reported that local tissue temperature increased by $0.8^{\circ} \mathrm{C}$ (Senova et al., 2017). Interestingly in that study, no effect was seen on neuronal cell death with acute light applications at the highest illumination used (Senova et al., 2017), however, the question still remains how the tissue can handle local heating with longer duration or repeated applications. If the conditions are right, tissue heating under illumination can cause damage and contribute to observed behavioral or physiological effects (Long and Fee, 2008). The use of nanoparticles made from inorganic scintillators will allow for the light source to be extremely close to ChR2 and therefore less light would be needed to activate ChR2, reducing the risk of damage caused by heat.

Here, we observed that light emitted from LSO:Ce microparticles in response to UV stimulation caused an increase in the frequency of sEPSCs onto CA1 pyramidal cells, indicating activation of ChR2. However, we did not detect firing of action potentials or large light-evoked EPSCs, suggesting that the activation of ChR2 is modest. UV light alone is also able to provide weak activation of ChR2, as seen by small photocurrents and an increase in sEPSC frequency. The photocurrents were modest with stimulation at $365 \mathrm{~nm}$, and even smaller at $315 \mathrm{~nm}$. Even though UV light activates ChR2, using the $315 \mathrm{~nm}$ wavelength improved our ability to detect events caused by light emission from the microparticles. One limitation of the current experiment is that the particles were applied to the surface of the slice, and therefore are likely to be 100 microns or more from 


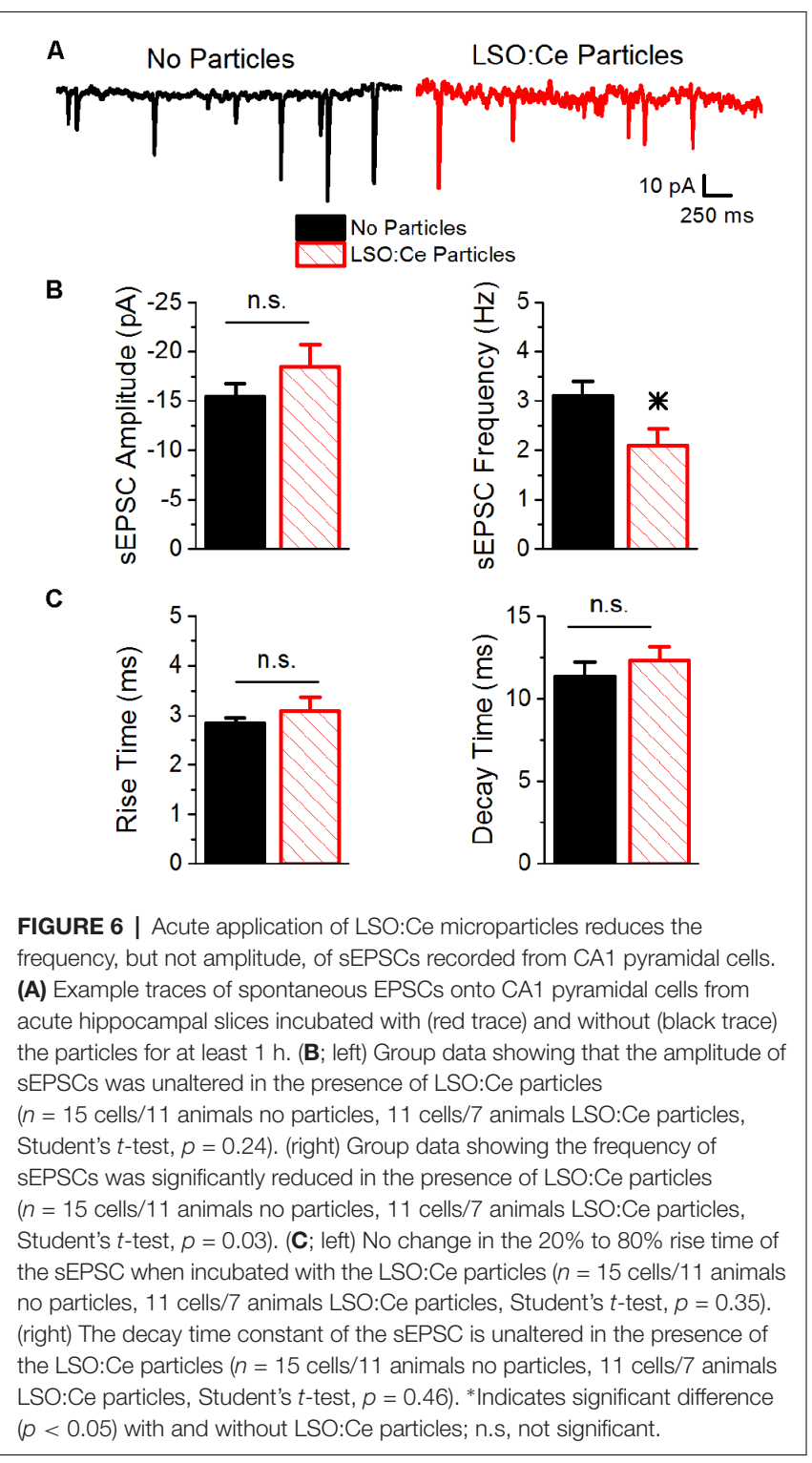

the cell expressing ChR2, which would partially account for the modest ChR2 activation observed. However, the proposed use of RLPs for in vivo optogenetics would use smaller particles that will be located much closer to $\mathrm{ChR} 2$, thus reducing light attenuation from scattering. As a result, the light needed for ChR2 activation in vivo should be even less.

We observed no toxicity in primary neuronal cultures following $24 \mathrm{~h}$ of LSO:Ce microparticle exposure, consistent with another study using coated LSO:Ce nanoparticles (Burdette et al., 2019). In acute hippocampal slices, there was no reactivity of the astrocytes as assessed by GFAP staining or decrease in the maximal strength of basal transmission assessed by I/O curves at CA3-CA1 synapses. However, there were still some minor effects on synaptic function. While there was no effect on sIPSCs, there was a small reduction in the frequency but not amplitude of sEPSCs together with an increase in PPR of evoked excitatory transmission, indicating an effect of

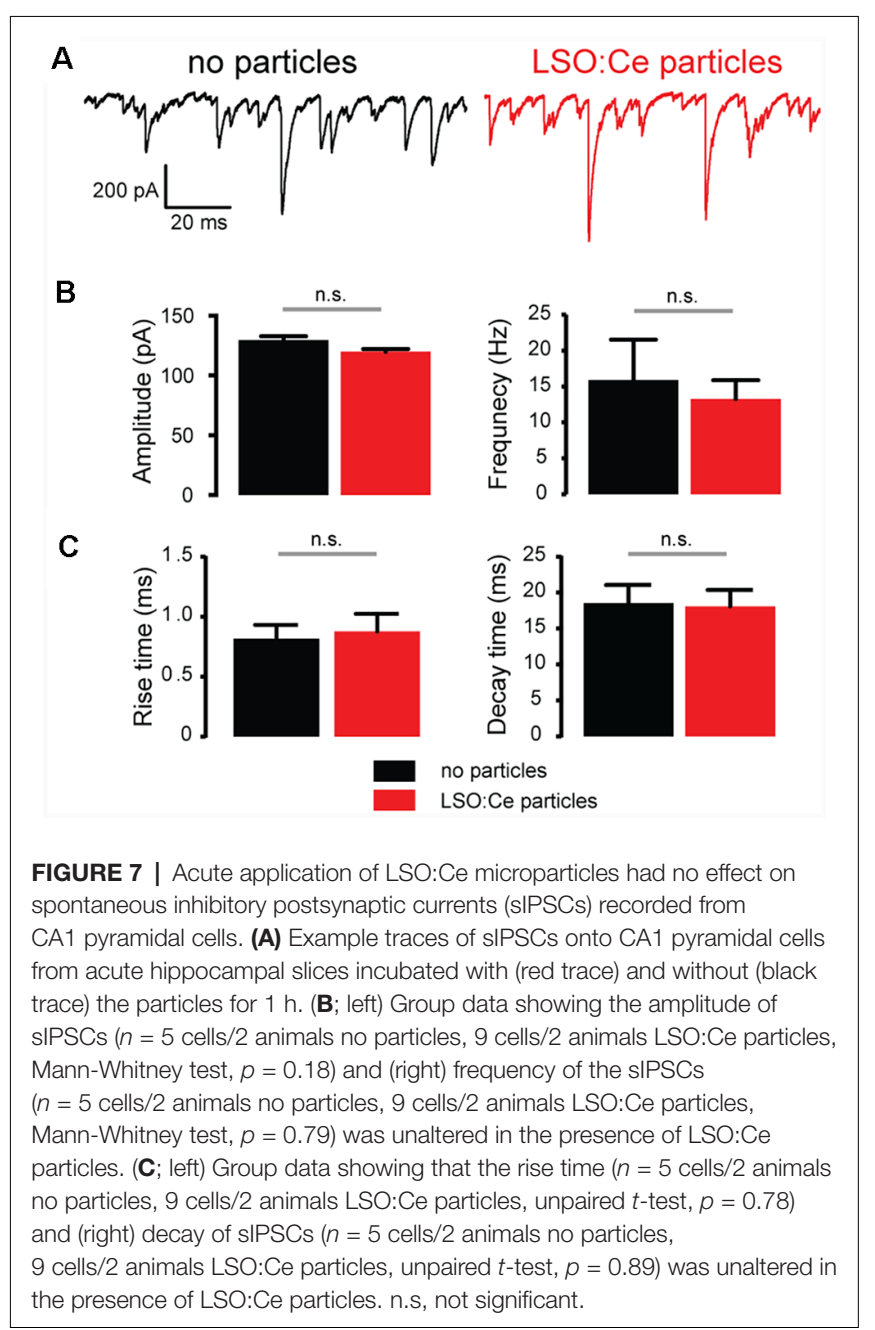

the particles on presynaptic function (Dobrunz and Stevens, 1997). There was also a small reduction in the input resistance of CA1 pyramidal cells, which could contribute to reduced cell excitability. Alterations in neuronal excitability have been shown to modulate presynaptic release probability (Crabtree et al., 2017). The axonal excitability was slightly diminished in the presence of the microparticles. Therefore, an alteration in presynaptic function that diminishes vesicular release probability could be the underlying mechanism for the minor effects on synaptic function with LSO:Ce microparticles exposure.

We also observed a slight but significant decrease in the magnitude of LTP, the long-term enhancement of synaptic strength and a cellular correlate of learning and memory (Malenka and Bear, 2004). Because LTP induction and expression is highly susceptible to cellular and behavioral stress, it is a sensitive measure of neuronal and synaptic health. LTP was induced using high-frequency stimulation and was monitored for 40 min post-tetanus. While the LSO:Ce particles did not affect the magnitude of the post-tetanic potentiation or the magnitude of LTP up to 20 min post-tetanus, there was a slight but significant decrease in the LTP magnitude at 40 min post-tetanus in the LSO:Ce treated slices. This suggests the possibility of a small 

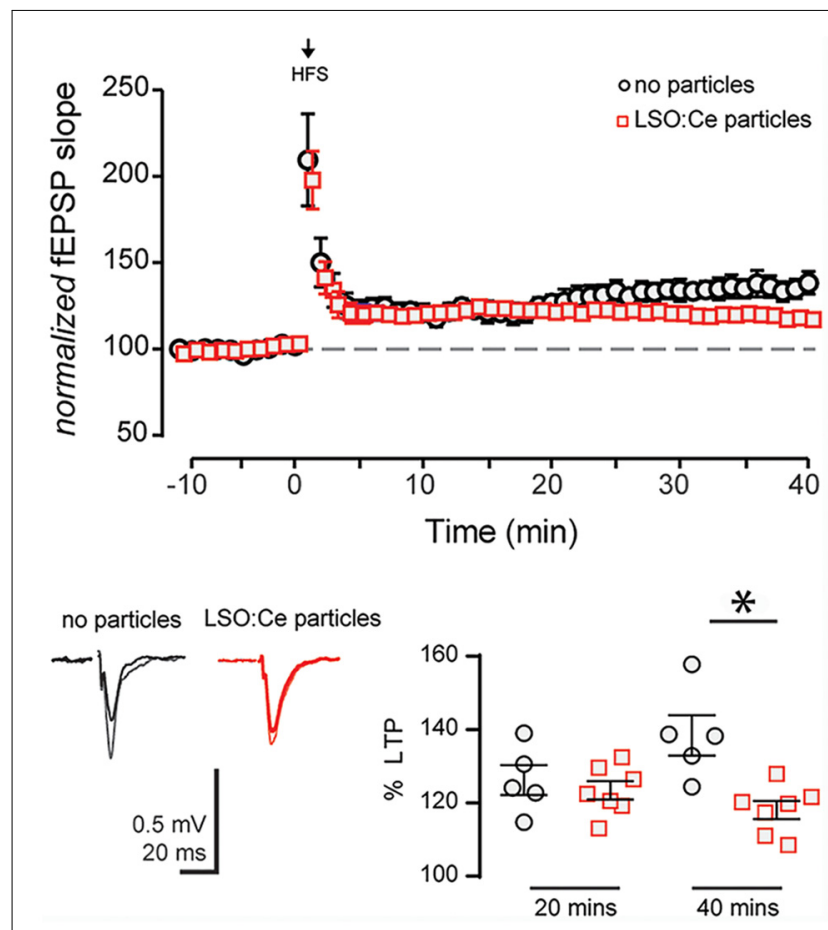

FIGURE 8 | Acute application of LSO:Ce microparticles decreases long term potentiation (LTP) at CA3-CA1 synapses. (A) The magnitude of High Frequency Stimulation (HFS, $5 \times 1 \mathrm{~s}$ at $100 \mathrm{~Hz}$ ) induced LTP at CA3-CA1 synapses was significantly decreased in slices incubated with particles.

(B) Averaged representative responses at -5 and +35 min to HFS stimulation (black and gray traces: no particles and red and light red traces: particles).

The deficit in the LTP magnitude did not occur until 30 min post HFS in slices incubated with particles for at least an hour (40 min LTP: $138 \pm 5 \%$ and $118 \pm 2 \%, n=5$ slices $/ 5$ animals no particles, $n=7$ slices $/ 7$ animals LSO:Ce particles, respectively; $p=0.0038 ; 20$ min LTP: $124 \pm 4 \%$ and $121 \pm 2 \%$, $n=5$ slices $/ 5$ animals no particles, $n=7$ slices $/ 7$ animals LSO:Ce particles, respectively; $\left.{ }^{*} p<0.05\right)$. Data represent mean \pm SEM. Significance determined by unpaired Student's $t$-test.

effect on the long-term expression of LTP, however, whether this change is biologically relevant to learning and memory is unknown.

Overall, the effects on synaptic and intrinsic neuronal properties were modest, indicating that the LSO:Ce particles do not majorly disrupt synaptic transmission and circuit function. Whether these minor effects on synaptic transmission are a result of physical interaction and the weight of the particles on the delicate neuronal structures is not clear. This study used particles that are larger $(4 \mu \mathrm{M})$ than would be used in vivo $(<100-200 \mathrm{~nm})$. It is possible that the effects of the smaller particles on synaptic transmission may be different, although they are unlikely to be greater. However, if toxic chemicals were

\section{REFERENCES}

Albertson, A. J., Bohannon, A. S., and Hablitz, J. J. (2017). HCN channel modulation of synaptic integration in gabaergic interneurons in malformed rat neocortex. Front. Cell. Neurosci. 11:109. doi: 10.3389/fncel.2017.00109

Andrabi, S. A., Umanah, G. K. E., Chang, C., Stevens, D. A., Karuppagounder, S. S., Gagné, J.-P., et al. (2014). Poly(ADP-ribose) polymerase-dependent energy leaching out of the particles to negatively impact cell health or if the weight of the particles was causing major cellular damage, we would likely have observed cell death in the primary neuronal cultures after $24 \mathrm{~h}$ of exposure. The GFAP staining is only able to detect gross changes in astrocyte morphology, therefore we cannot rule out small alterations in astrocytic processes in the presence of the microparticles. Additional effects on neuronal or astrocyte function or morphology might be observed with longer exposure times to the particles. Future studies are needed to determine if there are detrimental effects of LSO:Ce particles with UV or X-ray exposure potentially through the release of free radicals, as observed with cadmium-based quantum dots (Jamieson et al., 2007). However, any potential toxic effects might be overcome by PEGylation of the particles for in vivo use (Cheng et al., 2011).

Together, our results support the possibility of using LSO-Ce particles combined with ChR2 expression to non-invasively regulate synaptic circuit function in vivo.

\section{DATA AVAILABILITY}

The datasets generated for this study are available on request to the corresponding author.

\section{ETHICS STATEMENT}

Approval was obtained for all experimental protocols from the University of Alabama at Birmingham Institutional Animal Care and Use Committee. All experiments were conducted in accordance with the Guide for the Care and Use of Laboratory Animals adopted by the National Institutes of Health.

\section{AUTHOR CONTRIBUTIONS}

$\mathrm{LD}, \mathrm{LM}, \mathrm{AB}, \mathrm{KA}, \mathrm{LS}$, and SA designed the experiments. AB, KA, LS, DG, AK, and $\mathrm{MH}$ performed the experiments and analyzed the data. $\mathrm{LD}, \mathrm{LM}, \mathrm{AB}, \mathrm{KA}, \mathrm{SF}, \mathrm{MB}, \mathrm{MH}$, and SA wrote the initial draft, and $\mathrm{LD}, \mathrm{AB}$, and $\mathrm{LM}$ edited the manuscript.

\section{FUNDING}

This work was funded by NSF Track 2 FEC OIA-1632881 to LM and SF, and an Alabama State Funded EPSCoR Graduate Research Scholars Fellowship to KA.

\section{ACKNOWLEDGMENTS}

This manuscript has been released as a preprint at bioRXiv (Bartley et al., 2019; https://doi.org/10.1101/579722).

depletion occurs through inhibition of glycolysis. Proc. Natl. Acad. Sci. U S A 111, 10209-10214. doi: 10.1073/pnas.1405158111

Aravanis, A. M., Wang, L.-P., Zhang, F., Meltzer, L. A., Mogri, M. Z., Schneider, M. B., et al. (2007). An optical neural interface: in vivo control of rodent motor cortex with integrated fiberoptic and optogenetic technology. J. Neural Eng. 4, S143-S156. doi: 10.1088/1741-2560/ $4 / 3 / \mathrm{s} 02$ 
Barnett, S. C., Perry, B. A. L., Dalrymple-Alford, J. C., and Parr-Brownlie, L. C. (2018). Optogenetic stimulation: understanding memory and treating deficits. Hippocampus 28, 457-470. doi: 10.1002/hipo.22960

Bartley, A. F., Abiraman, K., Stewart, L. T., Hossain, M. I., Gahan, D. M., Kamath, A. V., et al. (2019). LSO:Ce inorganic scintillators are biocompatible with neuronal and circuit function. BioRxiv [Preprint]. doi: 10.1101/ 579722

Berndt, A., Schoenenberger, P., Mattis, J., Tye, K. M., Deisseroth, K., Hegemann, P., et al. (2011). High-efficiency channelrhodopsins for fast neuronal stimulation at low light levels. Proc. Natl. Acad. Sci. U S A 108, 7595-7600. doi: 10.1073/pnas.1017210108

Berry, R., Getzin, M., Gjesteby, L., and Wang, G. (2015). X-Optogenetics and U-Optogenetics: Feasibility and Possibilities. Photonics 2, 23-39. doi: $10.3390 /$ photonics 2010023

Burdette, M. K., Bandera, Y. P., Zhang, E., Trofimov, A., Dickey, A., Foulger, I., et al. (2019). Organic fluorophore coated polycrystalline ceramic LSO:Ce scintillators for X-ray bioimaging. Langmuir 35, 171-182. doi: 10.1021/acs. langmuir.8b03129

Canales, A., Park, S., Kilias, A., and Anikeeva, P. (2018). Multifunctional fibers as tools for neuroscience and neuroengineering. Acc. Chem. Res. 51, 829-838. doi: 10.1021/acs.accounts.7b00558

Chen, S., Weitemier, A. Z., Zeng, X., He, L., Wang, X., Tao, Y., et al. (2018). Near-infrared deep brain stimulation via upconversion nanoparticle-mediated optogenetics. Science 359, 679-684. doi: 10.1126/science.aaq1144

Cheng, Y., Zhao, L., Li, Y., and Xu, T. (2011). Design of biocompatible dendrimers for cancer diagnosis and therapy: current status and future perspectives. Chem. Soc. Rev. 40, 2673-2703. doi: 10.1039/c0cs00097c

Crabtree, G. W., Sun, Z., Kvajo, M., Broek, J. A. C., Fénelon, K., McKellar, H., et al. (2017). Alteration of neuronal excitability and short-term synaptic plasticity in the prefrontal cortex of a mouse model of mental illness. J. Neurosci. 37, 4158-4180. doi: 10.1523/jneurosci.4345-15.2017

Dissing-Olesen, L., and MacVicar, B. A. (2015). Fixation and immunolabeling of brain slices: SNAPSHOT method. Curr. Protoc. Neurosci. 71, 1.23.1-1.23.12. doi: 10.1002/0471142301.ns0123s71

Dobrunz, L. E., and Stevens, C. F. (1997). Heterogeneity of release probability, facilitation, and depletion at central synapses. Neuron 18, 995-1008. doi: 10.1016/s0896-6273(00)80338-4

Emiliani, V., Cohen, A. E., Deisseroth, K., and Häusser, M. (2015). Alloptical interrogation of neural circuits. J. Neurosci. 35, 13917-13926. doi: 10.1523/JNEUROSCI.2916-15.2015

Fenno, L. E., Gunaydin, L. A., and Deisseroth, K. (2015). Mapping anatomy to behavior in Thy1:18 ChR2-YFP transgenic mice using optogenetics. Cold Spring Harb. Protoc. 2015, 537-548. doi: 10.1101/pdb.prot 075598

French, D. N., Cannon, K., Bartley, A., McMahon, L., and Gray, G. (2018). "Novel $\mathrm{X}$-ray fluorescent organic monomer and polymer materials for optogenetic applications," in Biophotonics Congress: Biomedical Optics Congress 2018 (Microscopy/Translational/Brain/OTS), Washington, DC: USA, JTu3A.55.

Gorski, J. A., Talley, T., Qiu, M., Puelles, L., Rubenstein, J. L. R., and Jones, K. R. (2002). Cortical excitatory neurons and glia, but not GABAergic neurons, are produced in the Emx1-expressing lineage. J. Neurosci. 22, 6309-6314. doi: 10.1523/jneurosci.22-15-06309.2002

Gradinaru, V., Mogri, M., Thompson, K. R., Henderson, J. M., and Deisseroth, K. (2009). Optical deconstruction of parkinsonian neural circuitry. Science 324, 354-359. doi: 10.1126/science.1167093

Gunaydin, L. A., Grosenick, L., Finkelstein, J. C., Kauvar, I. V., Fenno, L. E., Adhikari, A., et al. (2014). Natural neural projection dynamics underlying social behavior. Cell 157, 1535-1551. doi: 10.1016/j.cell.2014.05.017

Hoque, A., Williamson, N. A., Ameen, S. S., Ciccotosto, G. D., Hossain, M. I., Oakhill, J. S., et al. (2019). Quantitative proteomic analyses of dynamic signalling events in cortical neurons undergoing excitotoxic cell death. Cell Death Dis. 10:213. doi: 10.1038/s41419-019-1445-0

Jamieson, T., Bakhshi, R., Petrova, D., Pocock, R., Imani, M., and Seifalian, A. M. (2007). Biological applications of quantum dots. Biomaterials 28, 4717-4732. doi: 10.1016/j.biomaterials.2007.07.014

Lempicki, A., Brecher, C., Lingertat, H., Miller, S. R., Glodo, J., and Sarin, V. K. (2008). A ceramic version of the LSO scintillator. IEEE Trans. Nucl. Sci. 55, 1148-1151. doi: 10.1109/tns.2007.914368
Lim, D. H., Ledue, J., Mohajerani, M. H., Vanni, M. P., and Murphy, T. H. (2013). Optogenetic approaches for functional mouse brain mapping. Front. Neurosci. 7:54. doi: $10.3389 /$ fnins. 2013.00054

Lin, J. Y. (2011). A user's guide to channelrhodopsin variants: features, limitations and future developments. Exp. Physiol. 96, 19-25. doi: 10.1113/expphysiol. 2009.051961

Lin, X., Wang, Y., Chen, X., Yang, R., Wang, Z., Feng, J., et al. (2017). Multiplexed optogenetic stimulation of neurons with spectrum-selective upconversion nanoparticles. Adv. Healthc. Mater. 6:17. doi: 10.1002/adhm.201770090

Long, M. A., and Fee, M. S. (2008). Using temperature to analyse temporal dynamics in the songbird motor pathway. Nature 456, 189-194. doi: $10.1038 /$ nature 07448

Madisen, L., Mao, T., Koch, H., Zhuo, J. M., Berenyi, A., Fujisawa, S., et al. (2012). A toolbox of Cre-dependent optogenetic transgenic mice for light-induced activation and silencing. Nat. Neurosci. 15, 793-802. doi: 10.1038/nn.3078

Malenka, R. C., and Bear, M. F. (2004). LTP and LTD: an embarrassment of riches. Neuron 44, 5-21. doi: 10.1016/j.neuron.2004.09.012

Melcher, C. L., and Schweitzer, J. S. (1992). Cerium-doped lutetium oxyorthosilicate: a fast, efficient new scintillator. IEEE Trans. Nucl. Sci. 39, 502-505. doi: 10.1109/23.159655

Miller, A. P., Shah, A. S., Aperi, B. V., Kurpad, S. N., Stemper, B. D., and Glavaski-Joksimovic, A. (2017). Acute death of astrocytes in blastexposed rat organotypic hippocampal slice cultures. PLoS One 12:e173167. doi: 10.1371/journal.pone.0173167

Ozden, I., Wang, J., Lu, Y., May, T., Lee, J., Goo, W., et al. (2013). A coaxial optrode as multifunction write-read probe for optogenetic studies in non-human primates. J. Neurosci. Methods 219, 142-154. doi: 10.1016/j.jneumeth.2013.06. 011

Podgorski, K., and Ranganathan, G. (2016). Brain heating induced by near-infrared lasers during multiphoton microscopy. J. Neurophysiol. 116, 1012-1023. doi: 10.1152/jn.00275.2016

Rein, M. L., and Deussing, J. M. (2012). The optogenetic (r)evolution. Mol. Genet. Genomics 287, 95-109. doi: 10.1007/s00438-011-0663-7

Rost, B. R., Schneider-Warme, F., Schmitz, D., and Hegemann, P. (2017). Optogenetic tools for subcellular applications in neuroscience. Neuron 96, 572-603. doi: 10.1016/j.neuron.2017.09.047

Roy, S., Lingertat, H., Brecher, C., and Sarin, V. (2013). Optical properties of anisotropic polycrystalline $\mathrm{Ce}^{+3}$ activated LSO. Opt. Mater. 35, 827-832. doi: 10.1016/j.optmat.2012.09.039

Schmidt-Kastner, R., Wietasch, K., Weigel, H., and Eysel, U. T. (1993). Immunohistochemical staining for glial fibrillary acidic protein (GFAP) after deafferentation or ischemic infarction in rat visual system: features of reactive and damaged astrocytes. Int. J. Dev. Neurosci. 11, 157-174. doi: 10.1016/07365748(93)90076-p

Selimbeyoglu, A., Kim, C. K., Inoue, M., Lee, S. Y., Hong, A. S. O., Kauvar, I., et al. (2017). Modulation of prefrontal cortex excitation/inhibition balance rescues social behavior in CNTNAP2-deficient mice. Sci. Transl. Med. 9:eaah6733. doi: $10.1126 /$ scitranslmed.aah6733

Senova, S., Scisniak, I., Chiang, C.-C., Doignon, I., Palfi, S., Chaillet, A., et al. (2017). Experimental assessment of the safety and potential efficacy of high irradiance photostimulation of brain tissues. Sci. Rep. 7:43997. doi: 10.1038/srep43997

Shuba, Y. M. (2019). Use of scintillator-base nanoparticles for in vivo control of light-sensitive bioactive molecules. US Patent No 10,183,081. Washington, DC: U.S. Patent and Trademark Office. Filed February 7, 2013 and Issued January 22, 2019.

Stujenske, J. M., Spellman, T., and Gordon, J. A. (2015). Modeling the spatiotemporal dynamics of light and heat propagation for in vivo optogenetics. Cell Rep. 12, 525-534. doi: 10.1016/j.celrep.2015.06.036

Tanaka, Y., Tanaka, Y., Furuta, T., Yanagawa, Y., and Kaneko, T. (2008). The effects of cutting solutions on the viability of GABAergic interneurons in cerebral cortical slices of adult mice. J. Neurosci. Methods 171, 118-125. doi: 10.1016/j.jneumeth.2008.02.021

Wanner, I. B., Anderson, M. A., Song, B., Levine, J., Fernandez, A., GrayThompson, Z., et al. (2013). Glial scar borders are formed by newly proliferated, elongated astrocytes that interact to corral inflammatory and fibrotic cells via STAT3-dependent mechanisms after spinal cord injury. J. Neurosci. 33, 12870-12886. doi: 10.1523/JNEUROSCI.2121-13.2013 
Wu, L. G., and Saggau, P. (1994). Presynaptic calcium is increased during normal synaptic transmission and paired-pulse facilitation, but not in longterm potentiation in area CA1 of hippocampus. J. Neurosci. 14, 645-654.

Yang, H., Yan, H., Li, X., Liu, J., Cao, S., Huang, B., et al. (2018). Inhibition of connexin 43 and phosphorylated NR2B in spinal astrocytes attenuates bone cancer pain in mice. Front. Cell. Neurosci. 12:129. doi: 10.3389/fncel.2018. 00129

Yizhar, O., Fenno, L. E., Prigge, M., Schneider, F., Davidson, T. J., O'Shea, D. J., et al. (2011). Neocortical excitation/inhibition balance in information processing and social dysfunction. Nature 477, 171-178. doi: $10.1038 /$ nature 10360
Conflict of Interest Statement: The authors declare that the research was conducted in the absence of any commercial or financial relationships that could be construed as a potential conflict of interest.

Copyright (c) 2019 Bartley, Abiraman, Stewart, Hossain, Gahan, Kamath, Burdette, Andrabe, Foulger, McMahon and Dobrunz. This is an open-access article distributed under the terms of the Creative Commons Attribution License (CC BY). The use, distribution or reproduction in other forums is permitted, provided the original author(s) and the copyright owner(s) are credited and that the original publication in this journal is cited, in accordance with accepted academic practice. No use, distribution or reproduction is permitted which does not comply with these terms. 